\title{
Efficacy and safety of onabotulinumtoxinA with standardized occupational therapy for treatment of pediatric upper limb spasticity: Phase III placebo-controlled randomized
}

\section{trial}

Rozalina Dimitrova $^{\mathrm{a}, *}$, Emily McCusker ${ }^{\mathrm{a}}$, Mark Gormley ${ }^{\mathrm{b}}$, Darcy Fehlings ${ }^{\mathrm{c}}$, Katharine E. Alter ${ }^{\mathrm{d}}$, Susan Greaves ${ }^{\mathrm{e}}$, Chengcheng Liu ${ }^{\mathrm{f}}$ and Mitchell F. Brin ${ }^{\mathrm{a}, \mathrm{g}}$

aAllergan, an AbbVie company, Irvine, CA, USA

${ }^{\mathrm{b}}$ Gillette Children's Specialty Healthcare, St Paul, MN, USA

${ }^{\mathrm{c}}$ Holland Bloorview Kids Rehab Hospital, Department of Paediatrics, Toronto, ON, Canada

${ }^{\mathrm{d}}$ Mount Washington Pediatric Hospital, Baltimore, MD, USA

${ }^{\mathrm{e}}$ The Royal Children's Hospital, Melbourne, Victoria, Australia

${ }^{\mathrm{f}}$ Allergan, an AbbVie company, Madison, NJ, USA

${ }^{\mathrm{g}}$ University of California, Irvine, CA, USA

Received 26 February 2021

Accepted 15 June 2021

\begin{abstract}
.
BACKGROUND: This is the first large study of onabotulinumtoxinA as treatment for pediatric upper limb spasticity.

OBJECTIVE: Evaluate efficacy and safety of a single treatment with onabotulinumtoxinA plus occupational therapy (OT). METHODS: In this registrational phase III, multinational study (NCT01603602), participants were randomized 1:1:1 to onabotulinumtoxinA $3 \mathrm{U} / \mathrm{kg} / \mathrm{OT}, 6 \mathrm{U} / \mathrm{kg} / \mathrm{OT}$, or placebo/OT. Primary endpoint was average change from baseline at weeks 4 and 6 in Modified Ashworth Scale-Bohannon (MAS) score. Secondary endpoints included Modified Tardieu Scale (MTS), Clinical Global Impression of Change (CGI) and functional Goal Attainment Scale (GAS).

RESULTS: 235 participants were randomized. At weeks 4 and 6, onabotulinumtoxinA groups had greater mean reductions in MAS (both $-1.9 ; p<0.001$ ) versus placebo $(-1.2)$. OnabotulinumtoxinA doses improved dynamic tone per MTS. Mean CGI at weeks 4 and 6 was unchanged in the overall population, but improved in a post hoc analysis of patients with a single affected upper limb (UL) muscle group (elbow or wrist). GAS score for passive goals was significantly higher for $6 \mathrm{U} / \mathrm{kg}$ versus placebo at week 12. Most AEs were mild/moderate in severity; overall incidence was similar between groups.

CONCLUSIONS: OnabotulinumtoxinA ( 3 and $6 \mathrm{U} / \mathrm{kg}$ ) was safe and effective in reducing upper limb spasticity in pediatric participants.
\end{abstract}

Keywords: Children, onabotulinumtoxinA, randomized clinical trial, spasticity, upper limb

\footnotetext{
*Address for correspondence: Rozalina Dimitrova, Neuroscience Clinical Development, Allergan, an AbbVie company,
} 


\section{Background}

Cerebral palsy (CP) is a childhood neurologic condition caused by brain injury before cerebral development is complete (Rosenbaum et al., 2007) and occurs in 1.4 to three of every 1000 live births (Australian Cerebral Palsy Register, 2018; Sellier et al., 2016; Stern, 2018). Approximately $90 \%$ of cases involve muscle spasticity (Beckung et al., 2007; Reid et al., 2011), a sensorimotor system disorder characterized by a velocity-dependent increase in muscle tone (Gart \& Adkinson, 2018), causing impairment of motor skills (Arnaud et al., 2008), pain (Arnaud et al., 2008; Parkinson et al., 2010), and reduced longitudinal muscle growth (Herskind et al., 2016). Non-pharmacological treatments for spasticity include physical therapy, occupational therapy (OT), orthotics, casting, and splinting. As poor grasp and dexterity have a profound impact on daily living, OT is essential (Novak et al., 2020; Wilton, 2003). Systemic pharmacological treatments are available (Delgado et al., 2010; Shamsoddini et al., 2014), but they lack specificity and can have side effects.

OnabotulinumtoxinA is approved in several countries for treatment of upper and/or lower limb spasticity in adults (Allergan, 2017; Allergan Ltd., 2018). Successful treatment of children with CP using onabotulinumtoxinA was first reported in the early 1990s (Cosgrove et al., 1994; Koman et al., 1993), but to date, data showing improvement in upper limb function were available from small studies only (Fehlings et al., 2010; Fehlings et al., 2000; Ferrari et al., 2014; Olesch et al., 2010). OnabotulinumtoxinA has recently been approved in the United States for treatment of upper and lower limb spasticity in children.

Here we report the results of a large registrational study that led to the approval of onabotulinumtoxinA for the treatment of upper limb spasticity in children. This study was the first to assess the efficacy and safety of onabotulinumtoxinA as a treatment for upper limb spasticity in a large pediatric population. The study evaluated the safety and efficacy of a single treatment in either the elbow or the wrist (and fingers) with onabotulinumtoxin A ( 3 or $6 \mathrm{U} / \mathrm{kg}$ ) plus OT compared with placebo plus OT. The study was specifically designed to fulfill the rigors required for registration purposes with the Food and Drug Administration. It was hypothesized that the efficacy of spasticity management would be more effective in children who were treated with onabotulinumtoxinA and standardized OT than those who received placebo and standardized OT.

Spasticity was measured using the Modified Ashworth Scale-Bohannon (MAS) and the Modified Tardieu Scale (MTS). Functional assessments were performed by the Goal Attainment Scale (GAS) and Clinical Global Impression of Change (CGI) to fully assess the impact of the objective changes in muscle tone and spasticity with regard to everyday activities and overall function.

\section{Methods}

This phase III, multicenter, randomized, doubleblind, placebo-controlled, parallel-group, 12-week trial (ClinicalTrials.gov, NCT01603602) was conducted at 40 centers in Canada, Hungary, Philippines, Poland, Russia, South Korea, Thailand, Turkey, and the United States between 12 July 2012 and 6 July 2017. The distribution of patients across the study centers is summarized in Supplementary Table 1.

\subsection{Participants}

Children $(2$ to $<17$ years old, weight $\geq 10 \mathrm{~kg} / 22 \mathrm{lb}$ ) were monoplegic, hemiplegic, or triplegic, and had spasticity of the upper limb involving the elbow and/or wrist flexor muscles (confirmed by the Hypertonia Assessment Tool (Marsico et al., 2017), with single-arm sparing [only one arm requiring treatment]) secondary to CP. Spasticity characteristics met at least one of the following at screening and day 1 visits: elbow flexor tone $\geq 2$ measured by MAS and elbow flexor muscle contracture $\leq 30$ degrees, and/or wrist flexor tone of $\geq 2$ with finger flexor tone of $\geq 1$ measured by MAS and at least neutral position for passive range of the wrist with fingers at maximum extension. Participants had demonstrable movement and attempted utilization of the study limb at screening. Participants receiving anti-spasticity, muscle relaxant, and anti-epileptic medications, or intrathecal baclofen must have been on a stable dose and regimen for $\geq 30$ days before the day 1 visit.

Key exclusion criteria were any medical condition that may have increased risk after exposure to botulinum toxin type A (e.g., muscular dystrophy, myasthenia gravis, Eaton-Lambert syndrome, amyotrophic lateral sclerosis, mitochondrial disease, or any other significant disease that might interfere with neuromuscular function), predominant dystonia ( $\geq$ level 3 on Barry Albright Dystonia scale (Stew- 
art et al., 2017)) of study limb at screening, history of surgical intervention of study limb (except tendon lengthening $>12$ months before day 1 visit), history of fracture in study limb within 2 years of day 1 visit, previous casting within 6 months of day 1 visit or dynamic splint within 3 months for spasticity of study limb or affected lower limb(s), planned surgery of any $\operatorname{limb}(\mathrm{s})$, and casting or dynamic splint (stretching or stabilization devices to help with range of motion, e.g., Dynasplint ${ }^{\circledR}$ or UltraFlex ${ }^{\circledR}$ ) for spasticity of study limb or affected lower limb(s) during the study.

\subsection{Treatment and study design}

Participants were randomized (1:1:1 via interactive voice or web response system) to onabotulinumtoxinA $3 \mathrm{U} / \mathrm{kg}$ (not to exceed $100 \mathrm{U}$ ), $6 \mathrm{U} / \mathrm{kg}$ (not to exceed $200 \mathrm{U}$ ), or placebo, all with OT. Blinding was maintained by an Independent Drug Reconstitutor at each site. Participants were stratified according to age ( $\leq 6 ;>6$ years), designated principal muscle group (elbow, wrist flexors), and baseline MAS score of principal muscle group $(=2 ;>2)$. Based on which had the higher MAS score, participants had either elbow or wrist flexors (including fingers) designated as the principal muscle group; only the principal muscle group was treated. If both had an MAS score $\geq 2$, per protocol, the elbow was designated principal muscle group. As the study progressed, in participants in whom the MAS scores of elbow and wrist were equal, the wrist was designated principal, to ensure at least $40 \%$ representation in each group. OnabotulinumtoxinA or placebo was injected as per Supplementary Table 2 using muscle localization techniques (electrical stimulation, sonography, and/or electromyography). Use of minimal or moderate sedation/analgesia in addition to local anesthesia was permitted.

All participants were provided with standardized, weekly OT, defined as "physical interventions performed by a licensed therapist (or equivalent per local regulations) to facilitate functional improvement in the upper extremities," and help participants achieve their identified functional goals through provision of a treatment plan tailored to the goals identified for each child/adolescent. A therapy manual created by expert occupational therapists and physiotherapists in the field of cerebral palsy was used as the basis for training provided to all therapists who were delivering intervention in the trial. During the initial evaluation, therapists used task analysis to assess baseline performance and to help identify constraints/enablers (within the child, the environment, or the task) to completion of goal(s). The age of the child/adolescent and their cognitive and functional levels were considered when developing the program. OT was administered from about 2 weeks before randomization through study duration. Sessions lasted approximately 1 hour and included active assisted elongation and strengthening (progressive, resistive activities, open chain activities, closed chain activities, functional strengthening), which were goal-directed and targeted at muscle groups to facilitate achievement of the goal developed on the GAS, and task-oriented functional therapy to practice specific, identified goals. Home programs were encouraged, but use of additional therapies and modalities was not permitted.

The primary endpoint was average change from baseline in MAS score of the principal muscle group at weeks 4 and 6 . The resistance encountered to passive stretch was graded from 0 (no increase in tone) to 4 (affected part[s] rigid in flexion or extension) (Bohannon \& Smith, 1987). MAS scores of $0,1,1+$, 2,3 , or 4 were coded as a grade of $0,1,2,3,4$, or 5 , respectively. The study did not employ a second or independent rater, but those performing the assessment were given extensive training on how to conduct the measure.

Secondary endpoints were average CGI by Physician at weeks 4 and 6 , average change from baseline of MAS of the finger flexor muscle group at weeks 4 and 6, functional GAS at weeks 8 and 12, and MTS of the principal muscle group (Haugh et al., 2006). GAS was used to evaluate change in goal achievement as it has good evidence as a valid, reliable and sensitive instrument (Palisano, 1993).

CGI (Guy, 1976) was performed before MAS and MTS to reduce the likelihood of influence by spasticity assessments. For the GAS, two functional goals one functional active goal (e.g. ability to use scissors) and one functional passive goal (e.g. pain/spasm, tolerance of orthotic devices, reduction in care needs) were established for each participant at week -2 by participant and family in consultation with physician and/or therapist, and were agreed to with investigators and confirmed at the day 1 visit. Detection of change was assessed at an individual level. Goals were specific, measurable, achievable and realistic, resource sensitive, and timed/quantified (SMART). After all levels of the goals were set, they remained the same throughout the study. Goal achievement was assessed by the physician at weeks 8 and 12 
taking into consideration input from the treating therapist, caregiver, and/or participant. GAS was assessed using a scale of -3 (worse than start) to +2 (improvements clearly exceeded defined therapeutic goal) for active and passive goals. Goals were scaled by setting the desired outcome (level 0) through discussion with child/family, following which the study therapist set the remaining levels, providing sufficient detail to allow objective assessment of goal attainment with no gaps/overlap between levels, considering the child's history, age, present status, and study treatment period. MTS was assessed as the difference between slow (R2) and fast (R1) range of motion and respective change from baseline to each posttreatment visit. Quality of Upper Extremity Skills Test (QUEST) outcomes were also reported; scores varied from below 0 to 100 , and the total test score was the sum of all domain scores divided by the number of tested domains. Adverse events (AEs) were recorded.

An AE was defined as any unfavorable and unintended sign (including an abnormal laboratory finding), symptom, or disease temporally associated with the use of investigational product, whether or not related to the product. At each visit, the investigator assessed adverse events by asking each patient/caregiver a general, non-directed question such as "How have you been feeling since the last visit?" Directed questioning and examination followed as appropriate to determine seriousness and severity (mild=easily tolerated, moderate $=$ discomfort, or severe $=$ incapacitating) . Relatedness was also assessed by the physician.

\subsection{Statistical analysis}

Enrollment of approximately 224 participants was needed to ensure at least 213 (71 per group) completed the study with $86 \%$ power (significance level 0.05 ) for an assumed change from baseline of average week 4 and 6 MAS of -0.5 (standard deviation [SD]: 0.98). The modified intent-to-treat (mITT) population included all randomized participants with a valid MAS baseline score and at least one postbaseline measurement at weeks 2, 4, or 6 for MAS of the principal muscle group and CGI by Physician. The safety population included all treated participants.

The primary endpoint was analyzed using mixedeffect model repeated measures (MMRM) at a significance level of 0.05 , which included baseline
MAS as a covariate and age, principal muscle group, treatment group, visit, treatment-by-visit interaction, study center, and previous botulinum toxin exposure as factors. An unstructured covariance matrix was used to model the covariance of within-patient measures. A gate-keeping approach was used to control the type I error rate for the primary endpoint of MAS. The comparison of onabotulinumtoxinA $3 \mathrm{U} / \mathrm{kg}$ versus placebo was performed only if the comparison for $6 \mathrm{U} / \mathrm{kg}$ was statistically significant. Sensitivity analyses were performed using an analysis of covariance (ANCOVA) model using observed data and data with multiple imputations for missing values. CGI by Physician was rated at weeks 2, 4, 6, 8, and 12. Descriptive statistics were presented by treatment group at each assessment and for the average of weeks 4 and 6. Data were summarized and analyzed by MMRM as well as ANCOVA at each assessment using observed cases. AEs and medical history were coded using the Medical Dictionary for Regulatory Activities (v20.0).

Participants could have spasticity both in the elbow and in the wrist/fingers, but under the confines of the study, only one muscle group was treated. A post hoc analysis was performed in participants with baseline MAS $<2$ for the untreated muscle group, based on the hypothesis that an untreated, non-principal muscle group with moderate/severe spasticity in the same limb as the treated principal muscle could have hindered the ability to detect CGI improvements.

\subsection{Ethics approval and consent to participate}

Study investigators obtained approval of the study protocol from a properly constituted Institutional Review Board (IRB) or Independent Ethics Committee (IEC) prior to study initiation. The study was conducted in conformance with the International Council for Harmonisation E6 guideline for Good Clinical Practices and the principles of the Declaration of Helsinki, or the laws and regulations of the country in which the research was conducted, whichever afforded the greater protection to the individual.

Written informed consent was obtained at the first study visit. Written minor assent was obtained in accordance with local laws and IRB/IEC requirements. Written documentation was obtained in accordance with the relevant country and local privacy requirements, where applicable. 


\section{Results}

\subsection{Participants}

In all, 292 participants were screened, 235 were randomized, and 234 received study treatment and were included in the safety and mITT analyses (onabotulinumtoxinA: $6 \mathrm{U} / \mathrm{kg}, n=77 ; 3 \mathrm{U} / \mathrm{kg}, n=78$; placebo, $n=79)$. Three participants discontinued the study (Fig. 1), two in the onabotulinumtoxinA $6 \mathrm{U} / \mathrm{kg}$ group (one $\mathrm{AE}$, one for personal reasons) and one in the placebo group (withdrew before receiving treatment) (Fig. 1). Baseline demographics and disease characteristics were similar across groups (Table 1). The average post-treatment duration was 92.0, 91.4, and 89.3 days for onabotulinumtoxinA $6 \mathrm{U} / \mathrm{kg}, 3 \mathrm{U} / \mathrm{kg}$, and placebo, respectively.

\subsection{Spasticity measures}

The average of week 4 and 6 MAS least squares (LS) mean change from baseline in the principal mus- cle group was improved in both onabotulinumtoxinA groups as well as the placebo group, and was significantly greater for both onabotulinumtoxinA groups versus placebo (Fig. 2a; Supplementary Table 3). LS mean differences in MAS score from baseline were statistically significantly greater for both doses of onabotulinumtoxinA versus placebo at all study visits (Fig. 2b; Supplementary Table 3). Sensitivity analysis results were consistent with the primary analysis. The proportion of responders achieving $\geq 1$ grade reduction from baseline in MAS score was statistically greater for onabotulinumtoxinA $3 \mathrm{U} / \mathrm{kg}$ versus placebo groups at all study visits and at weeks 4 to 8 for onabotulinumtoxinA $6 \mathrm{U} / \mathrm{kg}$ versus placebo (Fig. 3a; Supplementary Table 3). A statistically significant (reduction) improvement in MAS score from baseline at weeks 4 and 6 was observed for elbow and wrist muscle groups (Supplementary Table 4). While baseline spasticity was less severe in fingers, and changes from baseline at weeks 4 and 6 were not statistically significant, numerical improvements

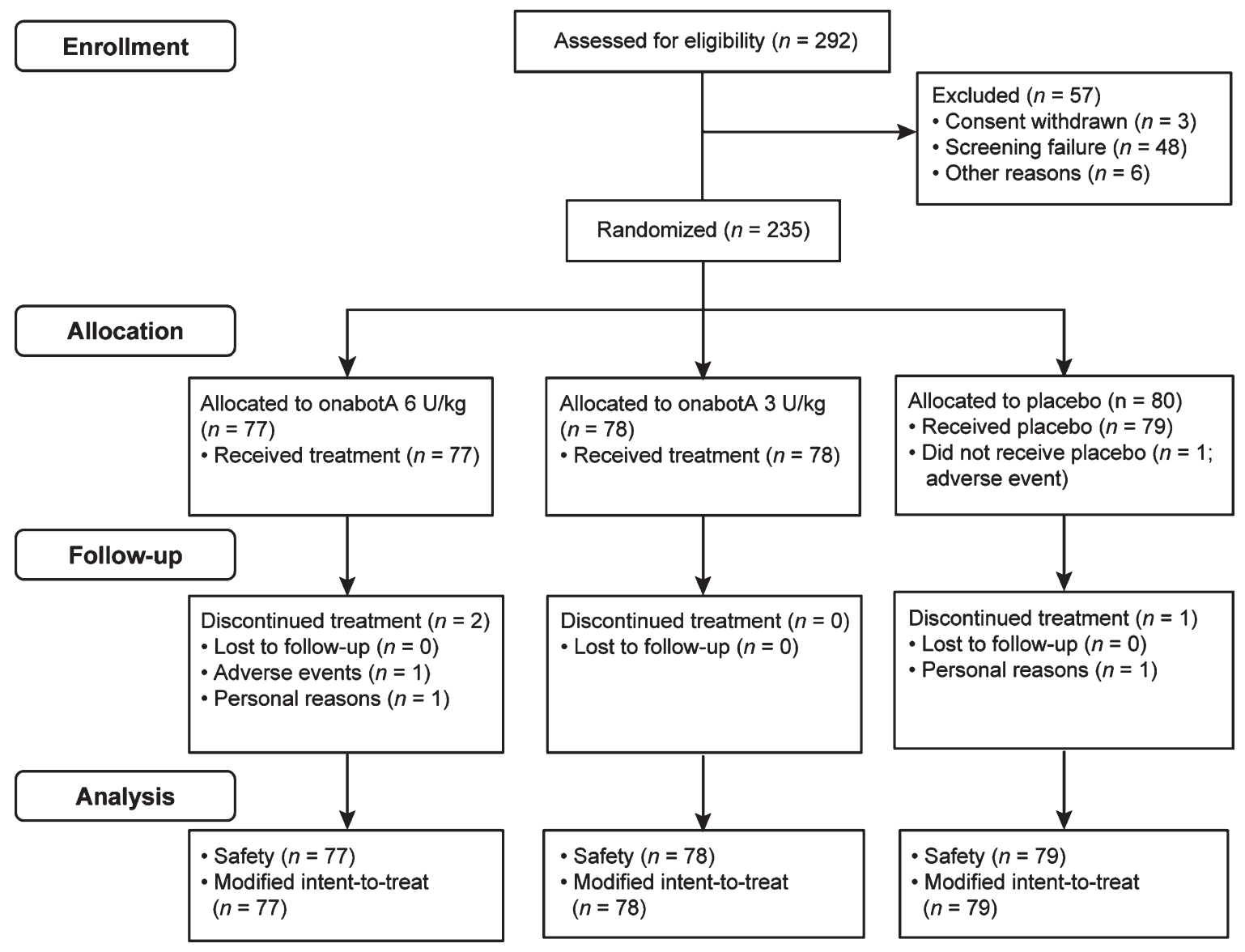

Fig. 1. CONSORT diagram. 
Table 1

Participant baseline and disease characteristics

\begin{tabular}{|c|c|c|c|}
\hline & \multicolumn{2}{|c|}{ OnabotulinumtoxinA } & \multirow[b]{2}{*}{ Placebo $n=79$} \\
\hline & $6 \mathrm{U} / \mathrm{kg} n=77$ & $3 \mathrm{U} / \mathrm{kg} n=78$ & \\
\hline \multicolumn{4}{|l|}{ Age, years } \\
\hline Mean $( \pm \mathrm{SD})$ & $7.6(3.66)$ & $8.3(4.48)$ & $7.8(4.06)$ \\
\hline$\leq 6$ years, $n(\%)$ & $35(45.5)$ & $33(42.3)$ & $34(43.0)$ \\
\hline \multicolumn{4}{|l|}{ Sex, $n(\%)$} \\
\hline Male & $50(64.9)$ & $42(53.8)$ & $47(59.5)$ \\
\hline \multicolumn{4}{|l|}{ Race, $n(\%)$} \\
\hline White & $51(66.2)$ & $42(53.8)$ & $51(64.6)$ \\
\hline Black & $3(3.9)$ & $3(3.8)$ & $3(3.8)$ \\
\hline Asian & $19(24.7)$ & $27(34.6)$ & $19(24.1)$ \\
\hline Hispanic & $2(2.6)$ & $4(5.1)$ & $5(6.3)$ \\
\hline Other & $2(2.6)$ & $2(2.6)$ & $1(1.3)$ \\
\hline GMFCS-E\&R, $n(\%)$ & $(n=77)$ & $(n=78)$ & $(n=80)$ \\
\hline Level I & $35(45.5)$ & $23(29.5)$ & $28(35.0)$ \\
\hline Level II & $27(35.1)$ & $32(41.0)$ & $42(52.5)$ \\
\hline Level III & $8(10.4)$ & $11(14.1)$ & $5(6.3)$ \\
\hline Level IV & $7(9.1)$ & $12(15.4)$ & $5(6.3)$ \\
\hline \multicolumn{4}{|l|}{ Principal muscle group, $n(\%)$} \\
\hline Elbow flexors & $48(62.3)$ & $48(61.5)$ & $48(60.8)$ \\
\hline Wrist flexors & $29(37.7)$ & $30(38.5)$ & $31(39.2)$ \\
\hline \multicolumn{4}{|l|}{ MAS of principal muscle group, $n(\%)$} \\
\hline 2 & $55(71.4)$ & $57(73.1)$ & $58(73.4)$ \\
\hline$>2$ & $22(28.6)$ & $21(26.9)$ & $21(26.6)$ \\
\hline \multicolumn{4}{|l|}{ Baseline MAS score (SD) } \\
\hline \multirow[t]{2}{*}{ Elbow flexors } & $(n=48)$ & $(n=48)$ & $(n=48)$ \\
\hline & $3.3(0.48)$ & $3.3(0.47)$ & $3.3(0.47)$ \\
\hline \multirow[t]{2}{*}{ Wrist flexors } & $(n=29)$ & $(n=30)$ & $(n=31)$ \\
\hline & $3.2(0.41)$ & $3.2(0.41)$ & $3.2(0.40)$ \\
\hline \multirow{2}{*}{ Finger flexors } & $(n=29)$ & $(n=30)$ & $(n=31)$ \\
\hline & $2.7(0.77)$ & $2.5(0.73)$ & $2.7(0.82)$ \\
\hline \multicolumn{4}{|l|}{ Disease type, $n(\%)$} \\
\hline Hemiplegia & $59(76.6)$ & $57(73.1)$ & $68(86.1)$ \\
\hline Monoplegia & 0 & 0 & 0 \\
\hline Triplegia & $18(23.4)$ & $21(26.9)$ & $11(13.9)$ \\
\hline \multicolumn{4}{|l|}{ Etiology, $n(\%)$} \\
\hline $\mathrm{CP}$ & $69(89.6)$ & $69(88.5)$ & $65(82.3)$ \\
\hline Stroke & $8(10.4)$ & $9(11.5)$ & $14(17.7)$ \\
\hline \multicolumn{4}{|l|}{ Previous botulinum toxin, $n(\%)$} \\
\hline No previous exposure & $32(41.6)$ & $33(42.3)$ & $34(43.0)$ \\
\hline Previous exposure for spasticity & $45(58.4)$ & $45(57.7)$ & $45(57.0)$ \\
\hline Mean time since first toxin exposure, days & 1443.7 & 1139.5 & 981.7 \\
\hline Mean time since last toxin exposure, days & 733.0 & 763.6 & 696.9 \\
\hline
\end{tabular}

CP: cerebral palsy, GMFCS-E\&R: Gross Motor Function Classification System - Expanded and Revised, MAS: Modified Ashworth Scale-Bohannon, SD: standard deviation.

were observed, with statistical significance at weeks 2 and 12 for $3 \mathrm{U} / \mathrm{kg}$ (Supplementary Table 4). Both onabotulinumtoxinA doses improved dynamic tone as measured by MTS. Differences were nominally significant for the $6 \mathrm{U} / \mathrm{kg}$ group at weeks 4 to 8 (elbow) and weeks 6 to 12 (wrist), and for the $3 \mathrm{U} / \mathrm{kg}$ group at week 4 (elbow) and weeks 2, 8, and 12 (wrist) (Supplementary Table 4). For finger flexors, differences in R1 (weeks 6 and 8) and R2 (weeks 2 to 8) were nominally significant at $6 \mathrm{U} / \mathrm{kg}$, although differences in R2-R1 were not.

\subsection{Functional measures}

A robust improvement in LS mean CGI score was observed with OT alone (placebo group). Although the average of weeks 4 and 6 CGI scores was not significantly greater in either onabotulinumtoxinA group (Supplementary Table 3), CGI scores were numerically improved in both onabotulinumtoxinA groups versus placebo at all study visits, with a nominal significant improvement for $3 \mathrm{U} / \mathrm{kg}$ at week 12 (Fig. 4). The proportion of responders with a CGI 
Table 2

Safety results

\begin{tabular}{|c|c|c|c|}
\hline & \multicolumn{2}{|c|}{ OnabotulinumtoxinA, $n(\%)$} & \multirow{2}{*}{$\begin{array}{c}\text { Placebo, } n(\%) \\
\quad(n=79)\end{array}$} \\
\hline & $6 \mathrm{U} / \mathrm{kg}(n=77)$ & $3 \mathrm{U} / \mathrm{kg}(n=78)$ & \\
\hline Overall AEs & $36(46.8)$ & $33(42.3)$ & $33(41.8)$ \\
\hline Treatment-related AEs & $7(9.1)$ & $7(9.0)$ & $2(2.5)$ \\
\hline Discontinuations due to AEs & $1(3.1)$ & 0 & 0 \\
\hline Serious AEs & $3(3.9)$ & $1(1.3)$ & $1(1.3)$ \\
\hline Treatment-related serious AEs & 0 & 0 & 0 \\
\hline Deaths & 0 & 0 & 0 \\
\hline \multicolumn{4}{|c|}{ AEs occurring in $\geq 2 \%$ of participants in any group (SOC/preferred term) } \\
\hline \multicolumn{4}{|c|}{ Infections and infestations } \\
\hline URI & $7(9.1)$ & $4(5.1)$ & $2(2.5)$ \\
\hline Viral URI & $6(7.8)$ & $4(5.1)$ & $5(6.3)$ \\
\hline Rhinitis & 0 & $3(3.8)$ & $1(1.3)$ \\
\hline Gastroenteritis viral & 0 & 0 & $2(2.5)$ \\
\hline \multicolumn{4}{|c|}{ General disorders and administration site conditions } \\
\hline Pyrexia & $3(3.9)$ & $3(3.8)$ & $5(6.3)$ \\
\hline Injection site pain & $3(3.9)$ & $2(2.6)$ & $1(1.3)$ \\
\hline \multicolumn{4}{|l|}{ Gastrointestinal disorders } \\
\hline Vomiting & $3(3.9)$ & $2(2.6)$ & $3(3.8)$ \\
\hline Diarrhea & 0 & $3(3.8)$ & $1(1.3)$ \\
\hline Nausea & $3(3.9)$ & 0 & 0 \\
\hline Constipation & $2(2.6)$ & 0 & $1(1.3)$ \\
\hline \multicolumn{4}{|c|}{ Musculoskeletal and connective tissue disorders } \\
\hline Muscular weakness & $1(1.3)$ & $3(3.8)$ & $1(1.3)$ \\
\hline Pain in extremity & $2(2.6)$ & $1(1.3)$ & $4(5.1)$ \\
\hline Arthralgia & 0 & $1(1.3)$ & $2(2.5)$ \\
\hline \multicolumn{4}{|c|}{ Respiratory, thoracic and mediastinal disorders } \\
\hline Cough & $2(2.6)$ & $1(1.3)$ & $3(3.8)$ \\
\hline Rhinorrhea & $3(3.9)$ & 0 & $1(1.3)$ \\
\hline Nasal congestion & $2(2.6)$ & 0 & $1(1.3)$ \\
\hline Epistaxis & 0 & $2(2.6)$ & 0 \\
\hline \multicolumn{4}{|l|}{ Nervous system disorders } \\
\hline Headache & $1(1.3)$ & $2(2.6)$ & $4(5.1)$ \\
\hline Seizure & $2(2.6)$ & $1(1.3)$ & 0 \\
\hline Partial seizures & $2(2.6)$ & 0 & 0 \\
\hline \multicolumn{4}{|c|}{ Injury, poisoning and procedural complications } \\
\hline Ligament sprain & 0 & $2(2.6)$ & $1(1.3)$ \\
\hline \multicolumn{4}{|c|}{ Reproductive system and breast disorders } \\
\hline Dysmenorrhea $^{\mathrm{a}}$ & 0 & $1(2.8)$ & 0 \\
\hline
\end{tabular}

${ }^{a}$ Denominator for percentage calculation was number of female participants $(n=36)$. AE: adverse event, SOC: System Organ Classe, URI: upper respiratory tract infection. Serious AEs occurred in three participants in the onabotulinumtoxinA $6 \mathrm{U} / \mathrm{kg}$ group (vomiting and pyrexia; seizure; infectious mononucleosis and stomatitis) and one participant each in the onabotulinumtoxinA $3 \mathrm{U} / \mathrm{kg}$ (meningitis) and placebo (osteochondrosis) groups. None was considered treatment-related.

score $\geq 1$ was higher in both onabotulinumtoxinA groups versus placebo at all study visits; differences were nominally significant for $6 \mathrm{U} / \mathrm{kg}$ at week 6 and for $3 \mathrm{U} / \mathrm{kg}$ at weeks 4 to 12 (Fig. 3b; Supplementary Table 3).

GAS by Physician score for passive goals was numerically higher in both onabotulinumtoxinA groups versus placebo at weeks 8 and 12 , and significantly higher $(p=0.003)$ for $6 \mathrm{U} / \mathrm{kg}$ at week 12 . No improvement in active goals was observed for onabotulinumtoxinA over placebo (Supplementary Table 5). QUEST total scores at week 12 were numer- ically, but not statistically, greater for both doses of onabotulinumtoxinA versus placebo (Supplementary Table 6).

An exploratory post hoc analysis was performed in participants with baseline MAS in the untreated nonprincipal muscle group of $<2$ (mild/no spasticity). The mean CGI averaged over weeks 4 and 6 was significantly higher for onabotulinumtoxinA $6 \mathrm{U} / \mathrm{kg}$ and $3 \mathrm{U} / \mathrm{kg}$ versus placebo. The difference was statistically significant in both onabotulinumtoxinA groups at all weeks except week 8 (Fig. 3c; Supplementary Table 3). 
(a)

OnabotulinumtoxinA $6 \mathrm{U} / \mathrm{kg}(n=77)$

OnabotulinumtoxinA $3 \mathrm{U} / \mathrm{kg}(n=78)$

Placebo $(n=79)$

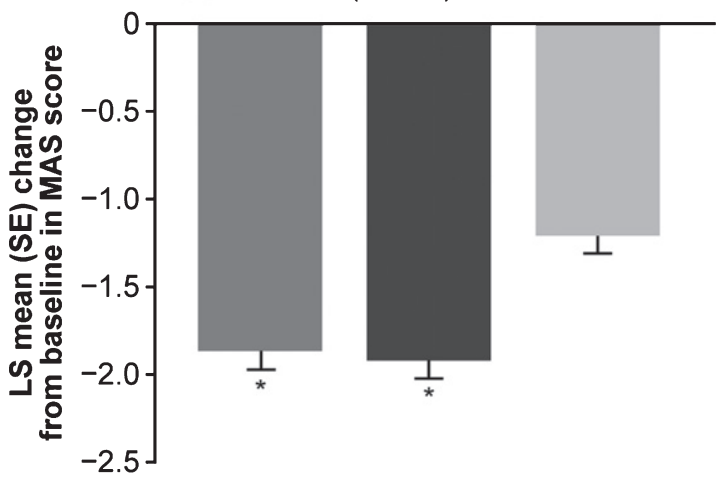

(b)
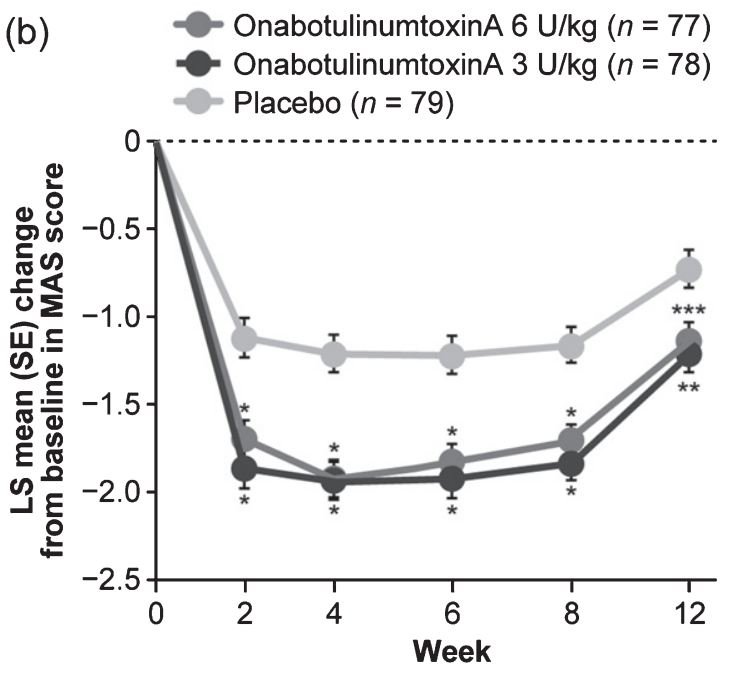

\begin{tabular}{cccc} 
& $\begin{array}{c}\text { OnabotulinumtoxinA } \\
6 \cup / \mathrm{kg}\end{array}$ & $\begin{array}{c}\text { OnabotulinumtoxinA } \\
3 \cup / \mathrm{kg}\end{array}$ & Placebo \\
\hline Baseline MAS & 3.3 & 3.3 & 3.3
\end{tabular}

Fig. 2. Change from baseline in MAS score of the principal muscle group. Change from baseline in MAS score a average at weeks 4 and 6 and $\mathbf{b}$ over the duration of the study. ${ }^{*} p<0.001,{ }^{* *} p=0.005$, ${ }^{* * *} p=0.001$ versus placebo. LS: least squares, MAS: Modified Ashworth Scale-Bohannon, SE: standard error.

\subsection{Safety measures}

Safety results are shown in Table 2. Most AEs were mild or moderate in severity and the overall incidence was similar for onabotulinumtoxinA and placebo groups. The only treatment-related AEs occurring in $\geq 1$ participant who received onabotulinumtoxinA were injection site pain ( $n=2$ in each dose group), muscular weakness (6U/kg: $n=1 ; 3 \mathrm{U} / \mathrm{kg}: n=3)$, and asthenia ( $n=1$ in each dose group). The only AEs occurring in $>3$ participants in any onabotulinum- (a)

OnabotulinumtoxinA $6 \mathrm{U} / \mathrm{kg}(n=77)$

OnabotulinumtoxinA $3 \mathrm{U} / \mathrm{kg}(n=78)$

Placebo $(n=79)$

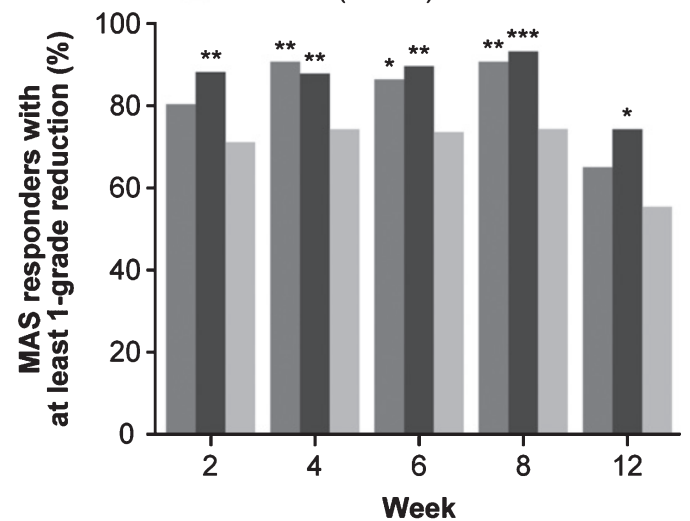

(b)

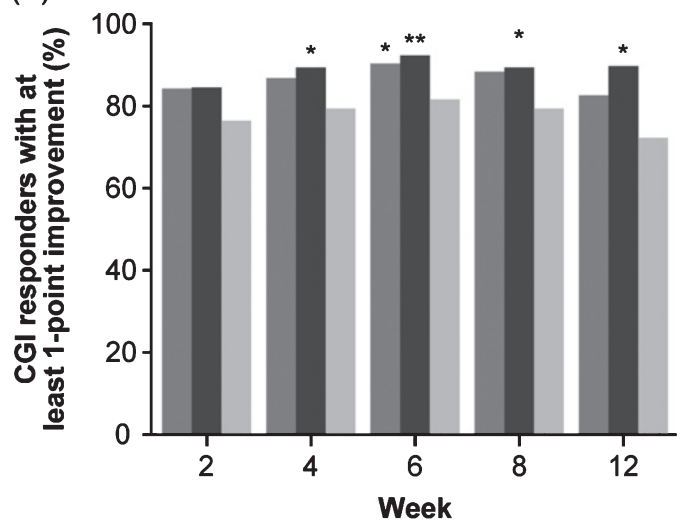

(c)

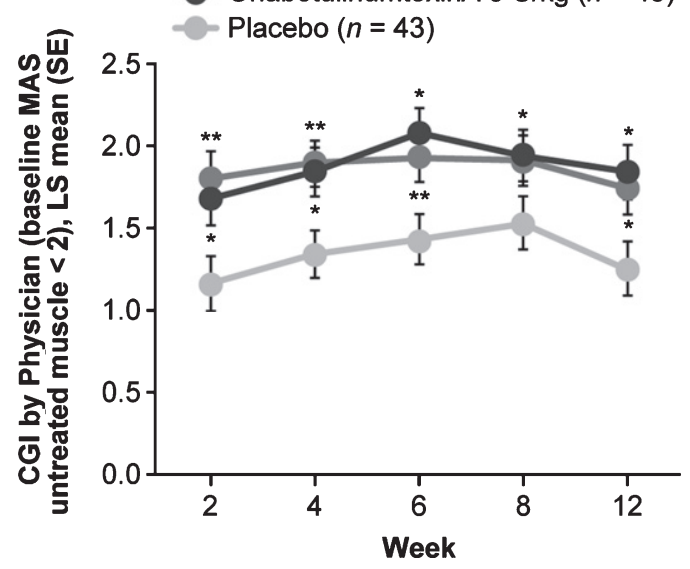

Fig. 3. Secondary efficacy outcomes. a) Proportion of responders according to MAS score. b) Proportion of responders according to CGI by Physician score. c) Post hoc analysis: CGI over time in participants with no or mild spasticity (baseline MAS $<2$ ) in the untreated non-principal muscle group. ${ }^{*} p<0.05,{ }^{* *} p<0.01$, ${ }^{* * *} p<0.001$ versus placebo. CGI: Clinical Global Impression, LS: least squares, MAS: Modified Ashworth Scale-Bohannon, SE: standard error. 


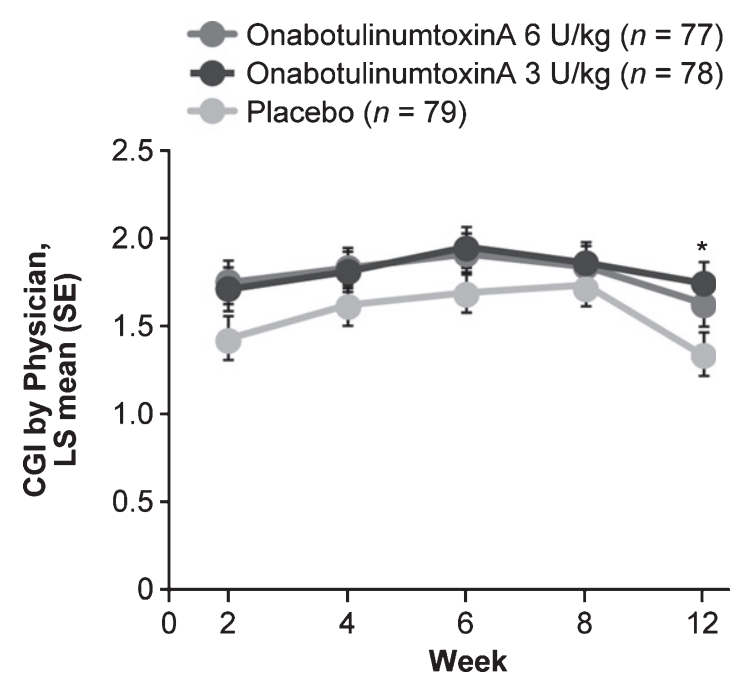

Fig. 4. CGI by visit. ${ }^{*} p<0.05$ vs placebo. CGI: Clinical Global Impression of Change, LS: least squares, SE: standard error.

toxinA group were upper respiratory tract infection (URI) and viral URI. The frequency of non-viral URI trended upward with dose. None was serious or severe, considered treatment-related, or resulted in study discontinuation. Three AEs of seizure $(6 \mathrm{U} / \mathrm{kg}$ : $n=2 ; 3 \mathrm{U} / \mathrm{kg}: n=1)$ and two AEs of mild partial seizure $(6 \mathrm{U} / \mathrm{kg})$ were reported in onabotulinumtoxinA groups. Seizure is a common CP comorbidity; medical history of seizure was documented in $35 \%$ $(82 / 234)$ of study participants. None of the reported seizure events was considered treatment-related; only one $(3 \mathrm{U} / \mathrm{kg})$ was new onset. One participant in the onabotulinumtoxinA $6 \mathrm{U} / \mathrm{kg}$ group discontinued the study due to an $\mathrm{AE}$ (moderate but serious case of stomatitis 108 days after treatment; unrelated to study treatment). There were no AEs deemed related to distant spread of toxin and no deaths.

\section{Discussion}

The results of this phase 3 , multicenter, randomized trial show that onabotulinumtoxinA/OT-treated participants had a robust and significant reduction from baseline in muscle tone (MAS) for the average of weeks 4 and 6 (primary endpoint), and at all study visits up to week 12 compared with placebo/OT. Similarly, improvements in MAS were observed for individual elbow, wrist, and finger muscle groups. The lack of clear dose-response suggests that $3 \mathrm{U} / \mathrm{kg}$ per muscle group (elbow or wrist/fingers) may be an appropriate starting dose in either elbow or wrist/finger muscle group. The SD of the average week 4 and 6 MAS change from baseline in the current study is approximately 1.0, which is comparable with the pooled SD (0.8) of MAS change from baseline at week 6 in adult upper limb studies (Brashear et al., 2002; Childers et al., 2004; Kaji et al., 2010) suggesting that data in children have similar variability as the adult data.

The beneficial effect of onabotulinumtoxinA on spasticity reduction was noted in both the MAS and the MTS. The CGI (weeks 4 and 6 average) was not significantly greater in either onabotulinumtoxinA group versus placebo. However, this may be attributed to the study design limitation wherein only one muscle group was treated. Despite the improvement seen with placebo/OT, mean CGI scores were further improved in both onabotulinumtoxinA groups at every study visit. The proportion of responders with a CGI score $\geq 1$ reached statistical significance for onabotulinumtoxinA versus placebo at several time points. Also, the MAS and CGI were strongly correlated, suggesting that the reduction in limb tone was clinically meaningful.

Data normality testing of the site-specific treatment effect indicated normal distribution of the MAS and CGI data at the primary time points (average of week 4 and 6). In addition, a box plot analysis identified one potential site outlier for MAS in the $6 \mathrm{U} / \mathrm{kg}$ group driven by a single patient, no outliers were identified for CGI. No sensitivity analyses were deemed warranted for the single outlier and we conclude that no individual sites influenced the overall study results.

The clear evidence of global improvement based on the CGI responder analysis in this study prompted a post hoc analysis. As the study allowed only a single muscle group to be treated, the analysis was performed on the subset of patients who did not require treatment in additional muscle groups in the treated limb. OnabotulinumtoxinA demonstrated statistically significant global improvements in these participants, suggesting that because both wrist and elbow muscle groups are used to perform daily tasks, untreated upper limb spasticity may hinder improvement and ability to detect global improvements.

OnabotulinumtoxinA/OT was superior to placebo/OT for passive, but not active, goals. This may be reflective of relevant passive goals such as splint tolerance being more readily achievable than active goals, or because of limiting onabotulinumtoxinA injection to one muscle group. Treating all affected muscles may improve active goal outcomes, but this was outside the scope of 
this study. Further, whereas participants with lower limb spasticity use muscles repetitively during weight-bearing locomotor tasks, thus providing naturalistic reinforcement of the effects of focal treatments, participants with hemiplegic upper limb spasticity often compensate by performing tasks with one hand (untreated), making retraining more challenging (Boyd et al., 2001). The relatively short duration of this single-treatment study may have been insufficient to achieve sufficient gains in active goals over those achieved with OT alone.

OnabotulinumtoxinA was well tolerated with a safety profile similar to placebo and consistent with previous studies. The main limitation of this study is that treatment was not consistent with the realworld setting in which multiple muscle groups or limb segments would be independently assessed and, if clinically indicated, treated.

\section{Conclusions}

The results of this registrational phase III study compared a single treatment with onabotulinumtoxinA $3 \mathrm{U} / \mathrm{kg}$ or $6 \mathrm{U} / \mathrm{kg}$ versus placebo, all in addition to OT, for the treatment of upper limb spasticity in children aged 2 to $<17$ years. Both onabotulinumtoxinA doses were well tolerated in this pediatric population. The study met its primary endpoint; both onabotulinumtoxinA doses demonstrated a statistically significant change from baseline in MAS (average weeks 4 and 6) versus placebo. Although the prespecified average of weeks 4 and 6 CGI score was not significantly greater for onabotulinumtoxinA versus placebo, significant global improvements were evident in the CGI responder analysis and in the subgroup of patients with spasticity in a single muscle group. Despite a lack of apparent dose-response in MAS outcomes, passive goals were improved with the $6 \mathrm{U} / \mathrm{kg}$ dose, suggesting a benefit of the higher dose for goal attainment. The benefits of onabotulinumtoxinA may be enhanced by adopting an approach that comprehensively treats the whole of the upper limb, and includes careful consideration of all affected muscles and targeted intervention to improve active function.

\section{Acknowledgments}

This study and its analyses were sponsored by Allergan plc, Dublin, Ireland. Editorial support for development of this manuscript was provided by Helen Jones, PhD, CMPP on behalf of Evidence Scientific Solutions, Inc, Philadelphia, PA, and was funded by Allergan plc, Dublin, Ireland.

\section{Conflict of interest}

RD, EM, CL, and MB are employees of Allergan, an Abbvie company, and hold stock and/or stock options in the company. MG is a consultant for Allergan, an Abbvie company and Ispen. DF has received research funding from Allergan, an AbbVie company. KEA has received honoraria from Haymarket Medical, Johns Hopkins University, and the North American Neurotoxin Association, and is a consultant/advisory board member for North American Neurotoxin Association and the International Neurotoxin Association. SG has received honoraria from Allergan, an Abbvie company.

\section{Supplementary data}

The supplementary tables are available from https://dx.doi.org/10.3233/NRE-210071.

\section{References}

Allergan. (2017). BOTOX (onabotulinumtoxinA) for injection, for intramuscular, intradetrusor, or intradermal use. Highlights of prescribing information. https://www.accessdata.fda.gov/drugsatfda_docs/label/2017/ 103000s5302lbl.pdf

Allergan Ltd. (2018). BOTOX ([OnabotulinumtoxinA] for injection, intramuscular, intradetrusor, or intradermal use). Summary of product characteristics. https://www.medicines.org.uk/emc/product/859/smpc

Arnaud, C., White-Koning, M., Michelsen, S. I., Parkes, J., Parkinson, K., Thyen, U., Beckung, E., Dickinson, H. O., Fauconnier, J., Marcelli, M., McManus, V., \& Colver, A. (2008). Parentreported quality of life of children with cerebral palsy in Europe. Pediatrics, 121(1), 54-64.

Australian Cerebral Palsy Register. (2018, February 25, 2019). Report of the Australian Cerebral Palsy Register: birth years 1995-2012. https://cpregister.com/wpcontent/uploads/2019/02/Report-of-the-Australian-CerebralPalsy-Register-Birth-Years-1995-2012.pdf

Beckung, E., Carlsson, G., Carlsdotter, S., \& Uvebrant, P. (2007). The natural history of gross motor development in children with cerebral palsy aged 1 to 15 years. Developmental Medicine and Child Neurology, 49(10), 751-756.

Bohannon, R. W., \& Smith, M. B. (1987). Interrater reliability of a modified Ashworth scale of muscle spasticity. Physical Therapy, 67(2), 206-207. 
Boyd, R. N., Morris, M. E., \& Graham, H. K. (2001). Management of upper limb dysfunction in children with cerebral palsy: a systematic review. European Journal of Neurology, 8(Suppl 5), 150-166.

Brashear, A., Gordon, M. F., Elovic, E., Kassicieh, V. D., Marciniak, C., Do, M., Lee, C. H., Jenkins, S., Turkel, C., \& Botox Post-Stroke Spasticity Study Group. (2002). Intramuscular injection of botulinum toxin for the treatment of wrist and finger spasticity after a stroke. $N$ Engl J Med, 347(6), 395-400.

Childers, M. K., Brashear, A., Jozefczyk, P., Reding, M., Alexander, D., Good, D., Walcott, J. M., Jenkins, S. W., Turkel, C., \& Molloy, P. T. (2004). Dose-dependent response to intramuscular botulinum toxin type A for upper-limb spasticity in patients after a stroke. Arch Phys Med Rehabil, 85(7), 1063-1069.

Cosgrove, A. P., Corry, I. S., \& Graham, H. K. (1994). Botulinum toxin in the management of the lower limb in cerebral palsy. Developmental Medicine and Child Neurology, 36(5), 386396.

Delgado, M. R., Hirtz, D., Aisen, M., Ashwal, S., Fehlings, D. L., McLaughlin, J., Morrison, L. A., Shrader, M. W., Tilton, A., \& Vargus-Adams, J. (2010). Practice parameter: pharmacologic treatment of spasticity in children and adolescents with cerebral palsy (an evidence-based review): report of the Quality Standards Subcommittee of the American Academy of Neurology and the Practice Committee of the Child Neurology Society. Neurology, 74(4), 336-343.

Fehlings, D., Novak, I., Berweck, S., Hoare, B., Stott, N. S., \& Russo, R. N. (2010). Botulinum toxin assessment, intervention and follow-up for paediatric upper limb hypertonicity: international consensus statement. European Journal of Neurology, 17(Suppl 2), 38-56.

Fehlings, D., Rang, M., Glazier, J., \& Steele, C. (2000). An evaluation of botulinum-A toxin injections to improve upper extremity function in children with hemiplegic cerebral palsy. Journal of Pediatrics, 137(3), 331-337.

Ferrari, A., Maoret, A. R., Muzzini, S., Alboresi, S., Lombardi, F., Sgandurra, G., Paolicelli, P. B., Sicola, E., \& Cioni, G. (2014). A randomized trial of upper limb botulimun toxin versus placebo injection, combined with physiotherapy, in children with hemiplegia. Research in Developmental Disabilities, 35(10), 2505-2513.

Gart, M. S., \& Adkinson, J. M. (2018). Considerations in the management of upper extremity spasticity. Hand Clinics, 34(4), 465-471.

Guy, W. (1976). ECDEU assessment manual for psychopharmacology. U.S. Department of Health, Education, and Welfare. https://archive.org/details/ecdeuassessmentm1933guyw/page/n1

Haugh, A. B., Pandyan, A. D., \& Johnson, G. R. (2006). A systematic review of the Tardieu Scale for the measurement of spasticity. Disability and Rehabilitation, 28(15), 899-907.

Herskind, A., Ritterband-Rosenbaum, A., Willerslev-Olsen, M., Lorentzen, J., Hanson, L., Lichtwark, G., \& Nielsen, J. B. (2016). Muscle growth is reduced in 15-month-old children with cerebral palsy. Developmental Medicine and Child Neurology, 58(5), 485-491.

Kaji, R., Osako, Y., Suyama, K., Maeda, T., Uechi, Y., Iwasaki, M., \& GSK Spasticity Study Group. (2010). Botulinum toxin type A in post-stroke upper limb spasticity. Curr Med Res Opin, 26(8), 1983-1992.
Koman, L. A., Mooney, J. F., 3rd, Smith, B., Goodman, A., \& Mulvaney, T. (1993). Management of cerebral palsy with botulinum-A toxin: preliminary investigation. Journal of Pediatric Orthopedics, 13(4), 489-495.

Marsico, P., Frontzek-Weps, V., Balzer, J., \& van Hedel, H. J. A. (2017). Hypertonia assessment tool: reliability and validity in children with neuromotor disorders. Journal of Child Neurology, 32(1), 132-138.

Novak, I., Morgan, C., Fahey, M., Finch-Edmondson, M., Galea, C., Hines, A., Langdon, K., Namara, M. M., Paton, M. C., Popat, H., Shore, B., Khamis, A., Stanton, E., Finemore, O. P., Tricks, A., Te Velde, A., Dark, L., Morton, N., \& Badawi, N. (2020). State of the evidence traffic lights 2019: systematic review of interventions for preventing and treating children with cerebral palsy. Current Neurology and Neuroscience Reports, 20(2), Article 3.

Olesch, C. A., Greaves, S., Imms, C., Reid, S. M., \& Graham, H. K. (2010). Repeat botulinum toxin-A injections in the upper limb of children with hemiplegia: a randomized controlled trial. Developmental Medicine and Child Neurology, 52(1), 79-86.

Palisano, R. J. (1993). Validity of goal attainment scaling in infants with motor delays. Phys Ther, 73(10), 651-658.

Parkinson, K. N., Gibson, L., Dickinson, H. O., \& Colver, A. F. (2010). Pain in children with cerebral palsy: a cross-sectional multicentre European study. Acta Paediatrica, 99(3), 446-451.

Reid, S. M., Carlin, J. B., \& Reddihough, D. S. (2011). Distribution of motor types in cerebral palsy: how do registry data compare? Developmental Medicine and Child Neurology, 53(3), 233-238.

Rosenbaum, P., Paneth, N., Leviton, A., Goldstein, M., Bax, M., Damiano, D., Dan, B., \& Jacobsson, B. (2007). A report: the definition and classification of cerebral palsy April 2006. Developmental Medicine and Child Neurology. Supplement, 109, 8-14.

Sellier, E., Platt, M. J., Andersen, G. L., Krägeloh-Mann, I., De La Cruz, J., Cans, C., \& Surveillance of Cerebral Palsy Network. (2016). Decreasing prevalence in cerebral palsy: a multi-site European population-based study, 1980 to 2003. Developmental Medicine and Child Neurology, 58(1), 85-92.

Shamsoddini, A., Amirsalari, S., Hollisaz, M. T., Rahimnia, A., \& Khatibi-Aghda, A. (2014). Management of spasticity in children with cerebral palsy [Review]. Iranian Journal of Pediatrics, 24(4), 345-351.

Stern, K. A. (2018). My Child at CerebralPalsy.org, an informational website owned by attorney Kenneth A. Stern: prevalence of cerebral palsy. Retrieved October 2018 from https://www.cerebralpalsy.org/about-cerebralpalsy/prevalence-and-incidence

Stewart, K., Harvey, A., \& Johnston, L. M. (2017). A systematic review of scales to measure dystonia and choreoathetosis in children with dyskinetic cerebral palsy. Developmental Medicine and Child Neurology, 59(8), 786-795.

Wilton, J. (2003). Casting, splinting, and physical and occupational therapy of hand deformity and dysfunction in cerebral palsy. Hand Clinics, 19(4), 573-584. 